\title{
Adaptive Scheduling for Wireless Video Transmission in High-Speed Networks
}

\author{
Zheng Wan ${ }^{1}$, Naixue Xiong ${ }^{2, *}$, Nasir Ghani ${ }^{3}$, Min Peng ${ }^{4}$, Athanasios V. Vasilakos ${ }^{5}$, Liang Zhou ${ }^{6}$ \\ ${ }^{1}$ School of Information Technology, Jiangxi Univ. of Finance \& Economics, China. cloudcity66@yahoo.com.cn \\ ${ }^{2}$ Department of Computer Science, Georgia State University, USA. nxiong@cs.gsu.edu \\ ${ }^{3}$ Department of Electrical \& Computer Engineering Univ. of New Mexico, USA.nghani@ece.unm.edu \\ ${ }^{4}$ Computer School of Wuhan University, China. pengm@whu.edu.cn \\ ${ }^{5}$ Department of Computer and Telecommunications, Engineering Univ. of Western Macedonia, Greece. vasilako@ath.forthnet.gr \\ ${ }^{6}$ UEI, ENSTA-ParisTech, Paris, France. Liang.zhou@ieee.org
}

\begin{abstract}
With the increase of wireless bandwidth, wireless video applications become more and more popular. A high speed core network is needed for large-scale applications to collect video from wireless terminals in a distributed way. It is necessary to guarantee video transmission in wireless networks because video data should be firstly transmitted via wireless channel. Since wireless bandwidth is still limited and fluctuates frequently, it is difficult to protect wireless video transmission. In this paper, we propose an adaptive cross layer scheduling schema to reduce video transmission distortion in IEEE 802.11e networks. Firstly, it is preferred to insert each video frame into the access category (AC) with minimum relative queuing delay to reduce transmission delay. Secondly, a dynamic frame assignment algorithm (DFAA) is proposed to guarantee the transmission of high priority frames efficiently, with the constraint of limited bandwidth. Finally, each parameter of DFAA is equipped with a fuzzy logic controller which can produce appropriate adjustment to reply quickly to the variation of video data rate, coding structure and network load. Extensive simulation results are presented to validate the effectiveness of our proposed scheme.
\end{abstract}

Keywords-Adaptive video transmission; High speed networks; Fuzzy logic; Cross layer; IEEE 802.11e

\section{INTRODUCTION}

Technical advances in high-speed networks have presented extremely wide bandwidth, encouraging significant increase in Internet traffic. In particular, with the development of wireless access and multimedia compression technologies, great attention has been devoted to wireless video communications. More and more users employed wireless terminals with camera and enjoyed various video applications through wireless access networks and core networks. Since the number of users increases quickly and video applications always have tremendous amount of data, it is required for high speed core networks. For example, a large-scale video surveillance application needs a fairly wide bandwidth to collect video captured by wireless cameras in different places so as to perform analysis in the server. Under such circumstances, video data should firstly be transmitted through wireless networks and then to the server via high speed core networks. Therefore, one of the key technologies of wireless video transmission in high speed networks is to guarantee video transmission quality in wireless access networks.
Up to now, two issues still stand to be resolved in this field. The first problem is how to optimize video communications over wireless links with limited bandwidth. Since video streams require stringent bandwidth, packet loss is inevitable. It is essential to identify the importance of video packets so as to ensure the transmission of high priority packets. Consequently, ideas of cross layer design [1] and unequal protection [2], which pose a bright foreground for video communications, were addressed. At the application layer, packet importance is calculated and packet priority is marked. At the network or MAC layer, unequal packet/frame scheduling is performed to reduce video transmission distortion.

The second problem is how to provide flexibility to the variation of video data rate, coding structure or network load. Solution of this problem requires cooperation between network scheduling algorithm and video encoding algorithm, i.e., cross layer optimization. For example, Navaratnam et al. [3] proposed to control transport layer offered load for multimedia streams based on the degree of medium contention information at MAC layer. Qin et al. [4] proposed a scheme which can adaptively select the number of enhancement layers according to available buffer size, transmission time and other parameters. The second problem is more complicated because capture and fast reply to the variation are difficult to provide.

As for wireless MAC standard, IEEE 802.11 shows its deficiency of not be capable of providing differentiated guarantees for different services. To satisfy the distinct Quality of Service requirements of multimedia service and data service, IEEE 802.11e [5] which is composed of Enhanced Distributed Channel Access (EDCA) and HCF Controlled Channel Access (HCCA) was proposed. Quality of Service support in EDCA is realized with the introduction of four access categories (ACs), among which AC2 is defined for video service. Each $\mathrm{AC}$ has its own transmission queue and a set of parameters to contend for transmission opportunities. However, this standard only provides a static mapping between service types and ACs.

To enhance its flexibility, several studies addressed different mapping ways. Ksentini et al. [6] proposed another static mapping algorithm for video streams using H.264 codec. Different types of H.264 video packets are mapped to different ACs. If applied to MPEG-4 codec, I, P and B frames could be

*Naixue Xiong is the corresponding author. 
categorized into $\mathrm{AC} 2, \mathrm{AC} 1$ and $\mathrm{AC} 0$ respectively. The performance of static mapping fluctuates as video coding structure and network load vary.

To overcome the deficiency of static mapping, C. H. Lin et al. [7] proposed a dynamic mapping algorithm for MPEG-4 streams, in which video frames are dynamically mapped to the appropriate $\mathrm{AC}$ based on the importance of video frame and network load. No matter which type a video frame belongs to, the algorithm always tries to assign it to AC2. A frame will be assigned to a lower priority $\mathrm{AC}$ with a dynamic calculated probability when congestion occurs. Obviously, less important video frame has a larger demotion probability. Beside, this work employs two queue length thresholds (threshold low and threshold_high) to identify network congestion level and to calculate demotion probability dynamically. Dynamic mapping, compared to EDCA and static mapping, reduces video transmission distortion and improves flexibility of IEEE $802.11 \mathrm{e}$ at a certain extent. However, this algorithm is not flexible to the variation of video data rate and coding structure. What is more, rules of choosing optimal parameters are not provided.

In this paper, an adaptive unequal protection scheme for video communications over IEEE 802.11e networks is addressed. Characteristics and contributions of this work are summarized in the following.

(1) We provide a relative queuing delay $\left(D_{R}\right)$ based $\mathrm{AC}$ selection mechanism. $D_{R}$ is an approximate value of actual queuing delay. Inserting video frame into the $\mathrm{AC}$ with minimum $D_{R}$ will reduce the transmission delay of each frame as well as overall distortion of the video stream.

(2) We integrate the $D_{R}$ based AC selection mechanism with a dynamic frame assignment algorithm (DFAA). DFAA takes video frame priority, $D_{R}$ and queue length (the number of packets in a queue) of each $\mathrm{AC}$ as inputs to differentiate frames with different priorities and to provide efficient and dynamic protection of video frames according to the real-time network load. Simulation results show that DFAA reduces video distortion significantly, compared with other reformed schemes.

(3) The fuzzy logic controller (abbr. as "FL controller") is designed to produce appropriate adjustment of DFAA parameter so as to provide flexibility to the variation of environments. An FL controller decides parameter adjustment according to queue length of certain $\mathrm{AC}$ and the frame loss rates of certain frame priorities. Experiments validate that DFAA with FL controller could achieve a near optimal performance when the DFAA parameter is initialized with an arbitrary value.

The rest of the paper is organized as follows. System framework and more details of our schema are introduced in Section II. Performance evaluation and discussions are provided in Section III. Finally, Section IV concludes the paper and points out future work.

\section{ADAPTIVE VIDEO TRANSMISSION SCHEME}

Firstly, abbreviations and symbols frequently used in this paper are presented in Table I and Table II.

\section{A. System Framework}

As shown in Fig. 1, the proposed scheme utilizes the capacities of $\mathrm{ACl}$ and $\mathrm{ACO}$ to improve video transmission performance. DFAA is the key component. Congestion level of each $\mathrm{AC}$ is recognized by queue length. Video frame priority, $D_{R}$ and queue length of each $\mathrm{AC}$, and parameter adjustment are collected to help DFAA to be aware of real-time network load so as to decide which AC it should throw the frame in. $D_{R}$ is calculated to represent actual queuing delay approximately. The FL controller takes statistical information within a time cycle (such as loss rates of video frames with different priorities) and queue length as inputs to determine quantitative adjustment of DFAA parameter for the next cycle.

Generally we use the term "frame" to refer to the frame of data link layer unless I, P, and B frames of MPEG-4 codec are discussed.

\section{B. Relative Queuing Delay}

To decrease transmission delay, a video frame should be assigned to the $\mathrm{AC}$ which has the shortest queuing delay. In IEEE 802.11e multi-queue model, frame queuing delay of an $\mathrm{AC}$ is proportional to the amount of queuing bytes and is inversely proportional to the scheduling opportunity of the AC. Although it is impossible to calculate the exact scheduling opportunities, the resulting average throughputs of different ACs within a long period under saturated circumstance can be adopted as their approximations. Let $B_{i}$ denote the amount of

TABLE I. ABBREVIATION DESCRIPTION

\begin{tabular}{l|l}
\hline Abbreviation & Description \\
\hline AC & Access Category \\
\hline DFAA & Dynamic Frame Assignment Algorithm \\
\hline EDCA & Enhanced Distributed Channel Access \\
\hline HCCA & HCF Controlled Channel Access \\
\hline FL & Fuzzy Logic \\
\hline ICM, Lin & Proposed scheme in [6] and [7] \\
\hline avgPSNR & Average PNSR \\
\hline \\
\multicolumn{2}{c}{ TABLE II. SYMBOL DEFINITIONS }
\end{tabular}

\begin{tabular}{l|l}
\hline Symbols & Description \\
\hline$D_{R}$ & Relative queuing delay \\
\hline$T_{2}: T_{1}: T_{0}$ & Ratio of average throughputs of AC2, AC1, AC0 \\
\hline $\mathrm{AC}_{\min }$ & The AC with the minimum $D_{R}$ \\
\hline $\mathrm{AC}_{\operatorname{mid}}$ & The AC with the middle $D_{R}$ \\
\hline $\mathrm{AC}_{\max }$ & The AC with the maximum $D_{R}$ \\
\hline$k_{1}, k_{2}, \ldots, k_{n}$ & Queue length thresholds of $\mathrm{AC}$ min and $\mathrm{AC}_{\text {mid }}$ \\
\hline$C_{j}$ & FL controller for $k_{j}$ \\
\hline$l_{j}$ & Loss rate of the th frame priority \\
\hline$R_{2}, R_{1}, R_{0}$ & Rate of traffic entering AC2, AC1 and AC0 \\
\hline$R_{v}, R_{b e}, R_{b g}$ & Rate of voice, best effort and background streams \\
\hline$R_{I}, R_{P}, R_{B}$ & Rate of I, $\mathrm{P}, \mathrm{B}$ video frames \\
\hline$P_{I}, P_{P}, P_{B}$ & Dropping probability of I, $\mathrm{P}, \mathrm{B}$ frames \\
\hline
\end{tabular}

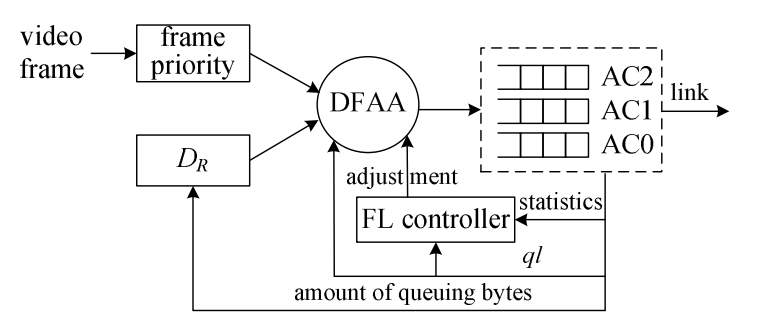

Figure 1. System framework of proposed scheme 
queuing bytes in $\mathrm{AC} i$ and let $T_{2}: T_{1}: T_{0}$ denote the ratio of average throughputs of $\mathrm{AC} 2, \mathrm{AC} 1$ and $\mathrm{AC} 0, D_{R}$ can be calculated as equation (1):

$$
D_{R i}=B_{i} / T_{i},
$$

where $D_{R i}$ denotes $D_{R}$ of $\mathrm{AC} i$. Notice that $B_{i}$ is a real-time parameter while $T_{2}: T_{1}: T_{0}$ could be determined in advance. Let $\mathrm{AC}_{\text {min }}, \mathrm{AC}_{\text {mid }}$ and $\mathrm{AC}_{\text {max }}$ denote the $\mathrm{AC}$ with the minimum, the middle and the maximum $D_{R}$ among three ACs respectively. Notice that $\mathrm{AC}_{\text {min }}, \mathrm{AC}_{\text {mid }}$ and $\mathrm{AC}_{\text {max }}$ vary in time.

\section{Dynamic Frame Assignment}

If we do not consider frame priority and try to insert each frame into $\mathrm{AC}_{\text {min }}$, different types of video frame will have the same dropping probability. Since distortion caused by the absence of high priority frames is more significant, DFAA performs unequal protection for different types of video frames to decrease the dropping probability of high priority frames. In DFAA the frame priority, $D_{R}$ and queue length of each AC are considered as scheduling parameters. The basic ideas are as follows: (1) Let $D_{R}$ identify the priority of an AC. AC priority is inversely proportional to $D_{R}$. If congestion level is not high, try to insert each video frame into the $\mathrm{AC}$ with the highest priority. (2) Let queue length denote the congestion level of an $\mathrm{AC}$. Define several thresholds for queue length of $\mathrm{AC}_{\text {min }}$ and $\mathrm{AC}_{\text {mid }}$, and match each threshold with a video frame priority. If real-time queue length is larger than the threshold correspondent to the frame priority, the video frame will be inserted into the $\mathrm{AC}$ with a lower priority.

DFAA scans $\mathrm{AC}_{\text {min }}$ and $\mathrm{AC}_{\text {mid }}$ in turn and determines whether a video frame could be inserted into one of them according to frame priority and real-time queue length of $\mathrm{AC}_{\text {min }}$ and $\mathrm{AC}_{\text {mid }}$. If both $\mathrm{ACs}$ are congested, the frame will be assigned to $\mathrm{AC}_{\max }$. DFAA is suitable for those video codecs who have limited priorities. To decrease the number of thresholds, $\mathrm{AC}_{\text {min }}$ and $\mathrm{AC}_{\text {mid }}$ can share thresholds. Assume a codec has $n+1$ priorities, then $n$ thresholds (denoted as $k_{1}$, $k_{2}, \ldots, k_{n}$; and $k_{1}>k_{2}>\ldots>k_{n}$ ) are required. Algorithm I describes the details of DFAA.

\section{Analysis of DFAA Parameters}

Predetermined parameters of DFAA include $T_{2}: T_{1}: T_{0}$ and queue length thresholds. $T_{2}: T_{1}: T_{0}$ can be determined through experiment approximately. In this experiment, standard EDCA is employed. We set the bandwidth of IEEE 802.11e wireless link to $1 \mathrm{Mbps}$. Data rate of voice is $64 \mathrm{kbps}$ (a rate commonly used) and data rates of the other three service types are $1 \mathrm{Mbps}$ respectively to produce a saturated link. The source, destination and packet length of various service types are uniform. From Table III we can find that the difference among various packet length settings is not distinct. We set $T_{2}: T_{1}: T_{0}$ to $9: 3: 1$, a near optimal value.

In DFAA the protection level of a certain frame priority is mainly determined by its opportunity of entering $\mathrm{AC}_{\text {min }}$. Thus the protection level of the highest priority is inversely proportional to the difference between the upper bound of queue length and $k_{1}$. And the protection level of the $j$ th priority is proportional to the difference between $k_{j}$ and $k_{j+1}$. Therefore, $k_{1}$ should be set to the upper bound of queue length to ensure the transmission of video frames with the highest priority. The optimal setting of $k_{j}(j>1)$ depends on the coding structure and the network load. For example, if the proportion of the $j$ th priority frames is large, it is necessary to increase $k_{j-} k_{j+1}$ and to decrease $k_{j-1}-k_{j}$. If the network load is very heavy, $k_{2}, k_{3}, \ldots, k_{n}$ should be set to a value near zero because maybe only the transmission of video frames with the highest priority could be ensured. On the contrary, $k_{2}, k_{3}, \ldots, k_{n}$ should be set to a value near the upper bound of queue length to take full advantage of available bandwidth.

During the transmission of VBR video streams over IEEE 802.11e networks, video data rate, coding structure and network load may vary dramatically, i.e. the optimal settings of $k_{2}, \ldots, k_{n}$ are dynamic. To find a near optimal setting, we address a fuzzy logic based self-adjusting scheme.

\section{E. FL Controller}

Each queue length threshold can be equipped with an FL controller. Since $k_{1}$ is always set to the upper bound of queue length, $n-1 \mathrm{FL}$ controllers $\left(C_{2}, \ldots, C_{n}\right)$ are required. $C_{j}$ is responsible for dynamic adjustment of $k_{j}$. Thus $C_{j}$ influences the transmission of video frames with the $j$-1th and the $j$ th priorities. Since the bandwidth of wireless links is limited, one controller will compete with the other controllers. To ensure the

Algorithm I: Dynamic Frame Assignment Algorithm (DFAA)

\section{1: Input:}

02: $B_{i}, i=0,1,2 ; T_{2}: T_{1}: T_{0}$; queue length of $\mathrm{AC}_{\text {min }}$ and $\mathrm{AC}_{\text {mid }}$

03: Priority of the video frame

04: Output:

05: AC assignment of the video frame

06: Procedure DynamicFrameAssignment

07 : for $(\mathrm{i}=0 ; \mathrm{i}<2 ; \mathrm{i}++)$

08: $D_{R i}=B_{i} / T_{i}$

09: Determine $\mathrm{AC}_{\text {min }}, \mathrm{AC}_{\text {mid }}$ and $\mathrm{AC}_{\text {max }}$ according to $D_{R i}$

10: switch \{priority\} \{

11: case 1:

12: if (queue length of $\mathrm{AC}_{\min }<k_{1}$ ) insert into $\mathrm{AC}_{\text {min }}$;

13: else if (queue length of $\mathrm{AC}_{\text {mid }}<k_{1}$ ) insert into $\mathrm{AC}_{\text {mid }}$;

14: else

15: break;

16: $\ldots \ldots \ldots . . . . .$.

17: case $j: / / 1<j<n+1$

18: if (queue length of $\mathrm{AC}_{\text {min }}<k_{j}$ ) insert into $\mathrm{AC}_{\text {min }}$;

19: else if (queue length of $\mathrm{AC}_{\text {mid }}<k_{j-1}$ ) insert into $\mathrm{AC}_{\text {mid }}$;

20: else

21: break;

22: $\quad \cdots \cdots \cdots . . . . .$.

23: case $n+1$ :

24: if (queue length of $\mathrm{AC}_{\text {min }}<k_{n}$ ) insert into $\mathrm{AC}_{\text {min }}$;

25: else if (queue length of $\mathrm{AC}_{\text {mid }}<k_{n}$ ) insert into $\mathrm{AC}_{\text {mid }}$;

26: else

27: break;

28: default

29: break; 30:

TABLE III. EXPERIMENT RESULT OF $T_{2}: T_{1}: T_{0}$

\begin{tabular}{c|c|c|c|c|c}
\hline $\begin{array}{c}\text { Packet } \\
\text { length(bytes) }\end{array}$ & $\begin{array}{c}\boldsymbol{T}_{\boldsymbol{2}} \\
(\mathbf{k b p s})\end{array}$ & $\begin{array}{c}\boldsymbol{T}_{\boldsymbol{1}} \\
\text { (kbps) }\end{array}$ & $\begin{array}{c}\boldsymbol{T}_{\boldsymbol{0}} \\
(\mathbf{k b p s})\end{array}$ & $\boldsymbol{T}_{\mathbf{2}} / \boldsymbol{T}_{\boldsymbol{1}}$ & $\boldsymbol{T}_{\boldsymbol{1}} / \boldsymbol{T}_{\boldsymbol{0}}$ \\
\hline 200 & 410.76 & 136.31 & 48.06 & 3.01 & 2.84 \\
\hline 500 & 532.59 & 161.15 & 64.30 & 3.31 & 2.51 \\
\hline 1000 & 581.94 & 188.42 & 66.38 & 3.09 & 2.84 \\
\hline
\end{tabular}


transmission of high priority frame, it is notable that FL controllers also have priorities. $C_{j}$ will not be activated unless $\left(C_{2}, \ldots, C_{j-1}\right)$ work well, i.e. the loss rates of the former $j-1$ frame priorities are decreased to zero. As for a specified controller $\mathrm{C}_{j}$, there are three inputs and one output.

(1) Input 1 : loss rate of the $j-1$ th priority, denoted as $l_{j-1}$.

(2) Input 2: loss rate of the $j$ th priority, denoted as $l_{j}$.

(3) Input 3: real-time queue length of $\mathrm{AC}_{\text {min }}$.

(4) Output: $k_{j}$ adjustment.

Considering implementation of $C_{j}$, triangular membership functions are employed for inputs. Both $l_{j-1}$ and $l_{j}$ have five levels (Low, Medium, High, Very high and Extremely high). Value ranges of $l_{j-1}$ and $l_{j}$ are divided unequally (level "Low" has the smallest range) to decrease loss rate more quickly. Queue length of $\mathrm{AC}_{\text {min }}$ has three levels, called Low, Medium and High. The output uses singleton membership function and has seven levels including three positive, zero and three negative adjustments. To raise the protection level of the $j$-1th priority, step size of negative adjustments is set to a larger value than that of positive adjustments.

Principles for setting fuzzy rules are as follows:

(1) Try to decrease $l_{j-1}$ and then try to decrease $l_{j}$ under the circumstance of $l_{j-1}=0$.

(2) The $k_{j}$ reduction is proportional to $l_{j-1}$ and the $k_{j}$ increment is proportional to $l_{j}$.

(3) The $k_{j}$ reduction is proportional to queue length of $\mathrm{AC}_{\min }$ and the $k_{j}$ increment is inversely proportional to queue length of $\mathrm{AC}_{\text {min }}$.

\section{PERFORMANCE EVALUATION}

Simulations are based on the integrated platform of ns-2 [8] and Evalvid [9], implemented by Chih-Heng Ke [10]. Network topology is presented in Fig. 2, in which wireless nodes $w 0 \sim w 3$ are connected to AP via IEEE 802.11e link. Foreman (300 frames, 10 seconds) and football (176 frames, 6 seconds) with MPEG-4 codec and CIF resolution are adopted as test sequences. The frame rate is set to 30 frames per second. As Table IV shows, data rates of two sequences at each second are quite different. From Table $\mathrm{V}$ we can also find the difference of coding structures between these two sequences, i.e. foreman has more I frames and B frames with respect to football. Since there are three types of video frames, two queue length thresholds and one FL controller should be deployed accordingly. The FL controller is responsible for adjusting $k_{2}$ to reduce dropping probability of both $\mathrm{I}$ frames and $\mathrm{P}$ frames. Bandwidth of IEEE 802.11e link is set to $4 \mathrm{Mbps}$.

In the first experiment, the performance of four schemes is studied: (1) standard EDCA; (2) static mapping [6], denoted by ICM; (3) dynamic mapping proposed by C. H. Lin [7], denoted by Lin; and (4) DFAA without FL controller. Two queue length thresholds of DFAA and Lin are both set to 50 and 25 . Prob_I, Prob_P, Prob_B of Lin are set to $0,0.3$ and 0.6 respectively. To make a comprehensive comparison, video sequences are transmitted under the following three scenarios.

(1) Streams of four service types are transmitted from $w 0 \sim w 3$ to $B$ respectively.

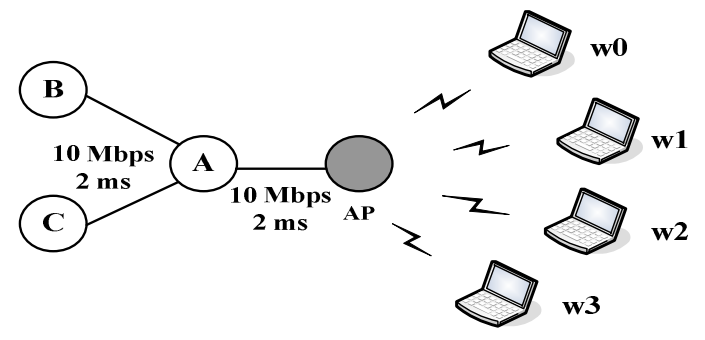

Figure 2. Simulation topology

TABLE IV. DATA RATES OF TWO SEQUENCES AT EACH SECOND

\begin{tabular}{c|l|l|c|l}
\hline $\begin{array}{c}\text { Second } \\
\text { No. }\end{array}$ & $\begin{array}{l}\text { Data rate } \\
\text { (foreman) }\end{array}$ & $\begin{array}{l}\text { Data rate } \\
\text { (football) }\end{array}$ & $\begin{array}{c}\text { Second } \\
\text { No. }\end{array}$ & $\begin{array}{l}\text { Data rate } \\
\text { (foreman) }\end{array}$ \\
\hline 1 & 2209.54 & 3368.70 & 7 & 2573.90 \\
\hline 2 & 1997.00 & 3342.50 & 8 & 2594.45 \\
\hline 3 & 2102.40 & 1727.28 & 9 & 2924.42 \\
\hline 4 & 2111.65 & 1713.87 & 10 & 2723.00 \\
\hline 5 & 2181.50 & 2448.97 & & \\
\hline 6 & 2322.80 & 2669.00 & & \\
\hline
\end{tabular}

TABLE V. TOTAL BYTES OF I/P/B FRAMES (a) foreman, scenario 1

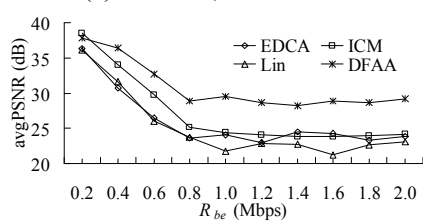

(c) foreman, Scenario 3

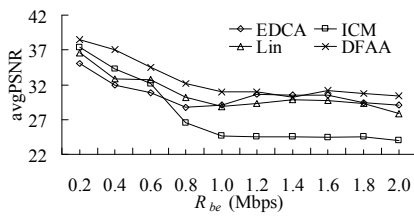

(e) football, scenario 2

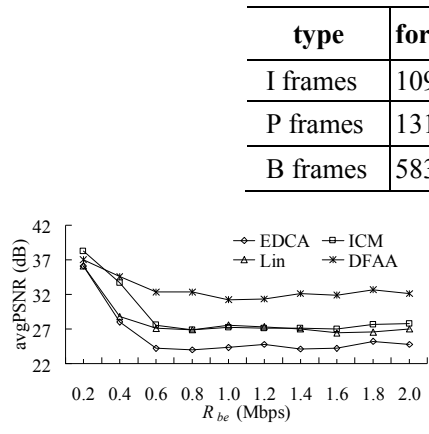

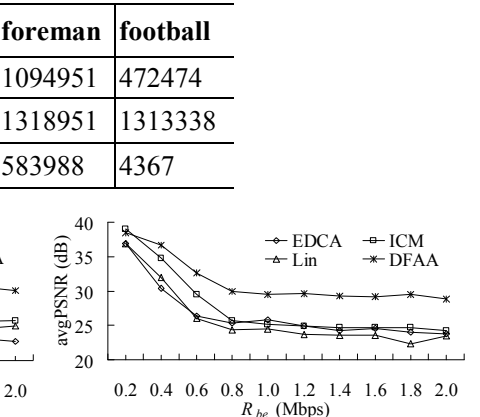

(b) foreman, scenario 2

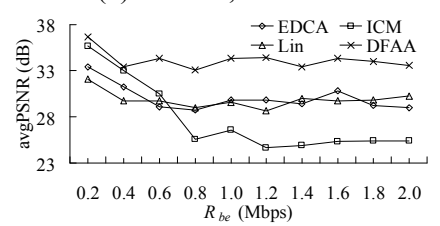

(d) football, scenario 1

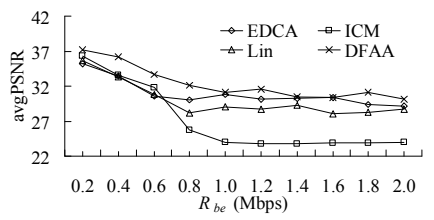

(f) football, scenario 3
Figure 3. Average PSNR comparison of two sequences in three scenarios

(2) Four streams are uniformly transmitted from $w 1$ to $B$.

(3) Streams are transmitted from $B$ to $w 0 \sim w 3$ respectively.

Since the decoding effects of I, P and B frames are quite different, the overall frame loss rate can not reflect video transmission distortion. Thus average PSNR is employed as the unique metrics in our evaluation.

Fig. 3 shows the average PSNR (avgPSNR) variation as data rates of best effort $\left(R_{b e}\right)$ and background traffic $\left(R_{b g}\right)$ increase. Notice that $R_{b e}: R_{b g}$ remains to 2 . From this figure we 
can find that avgPSNR of four schemes go down dramatically at first and then turn steady as $R_{b e}$ and $R_{b g}$ increase in every case. As we know video frames will occupy scheduling opportunities of $\mathrm{BE}$ and $\mathrm{BG}$ streams especially when $R_{b e}$ and $R_{b g}$ are low. Thus the number of video frames entering $\mathrm{AC} 1$ and AC0 reduces as $R_{b e}$ and $R_{b g}$ increase. However, effect of increasing $R_{b e}$ and $R_{b g}$ continuously after they reach the capacity of AC1 and AC0 is not notable because almost all increasing frames are dropped.

Table VI gives the average avgPSNR improvements of DFAA compared to other schemes in each case, in which we can find that DFAA reduces the transmission distortions of both sequences greatly. Fig. 3 and Table VI verify the analysis that existing schemes are not adaptive to the variation of environments. EDCA has a good performance when $R_{I}$ is low (football) and ICM is suitable for the case in which $R_{I}$ is high (foreman). Also the performance of ICM with football sequence is not bad when $R_{b e}$ and $R_{b g}$ are relatively low. On the other hand, it is difficult for Lin to achieve better performance with respect to EDCA and ICM. Table VII-VIII, which depict packet loss numbers of different frame type in two cases when $R_{b e}=1 \mathrm{Mbps}$, presents the reason. Lin always has the lowest overall packet loss rate, but significant $P_{I}$ increment degrades its performance. Although $P_{I}$ of ICM in both cases are 0 , too many $\mathrm{P}$ and $\mathrm{B}$ frames are dropped. DFAA achieves a good balance between $P_{I}$ and $P_{P} / P_{B}$.

Experiments below are all performed in scenario 1. From Fig. 4 we can find that $k_{2}=25$ is not suitable for every case. To achieve better performance, $k_{2}$ should be set to 10 when foreman is transmitted in a $3 \mathrm{Mbps}$ wireless link while it should be set to 45 for football in a $4 \mathrm{Mbps}$ link. The results verify the analysis that fixed parameter is not flexible to the variation of data rate, coding structure and network load. Furthermore, despite the issue of which value should $k_{2}$ be set to, the performance difference when applying various $k_{2}$ is remarkable. The maximum differences between two $k_{2}$ settings when $R_{b e}=2 \mathrm{Mbps}$ in four cases are 2.25, 0.74, 4.25 and 4.23.

Then we pay attention to the performance of DFAA with FL controller (abbr. as "DFAA-FL"). Since DFAA-FL could adjust $k_{2}$ according to the variation of environments, it is expected to provide the following advantages: (1) Achieve a near optimal performance; (2) Have a steady performance no matter which initial value is employed for $k_{2}$, so that $k_{2}$ can be initialized with an arbitrary value.

In this experiment, the adjustment cycle is set to 0.5 second while $R_{b e}$ is set to $2 \mathrm{Mbps}$. Table IX shows the avgPSNR variation of DFAA-FL when $k_{2}$ is initialized with different values. Unlike the results of Fig. 4, the performance of DFAAFL keeps relatively steady in each case as initial value of $k_{2}$ varies. It is notable that in some cases (case 1 and case 2) the performance of DFAA-FL may be better than any solution with fixed $k_{2}$ in Fig. 4. Also the performance may be close to the best one among DFAA-10, DFAA-25 and DFAA-45 in other cases (case 3 and case 4), indicating that DFAA-FL can achieve a near optimal performance.

Finally, Fig. 5 shows the adjustment details of $k_{2}$ as the simulation goes on when deployed with different initial value. From the figure we find that the variation of $k_{2}$ depends on link
TABLE VI. AVERAGE AVGPSNR IMPROVEMENT OF DFAA

\begin{tabular}{l|l|l|l|l|l|l}
\hline & $\begin{array}{c}\text { foreman } \\
\text { scenario 1 1 }\end{array}$ & $\begin{array}{c}\text { foreman } \\
\text { scenario 2 }\end{array}$ & $\begin{array}{c}\text { foreman } \\
\text { scenario 3 3 }\end{array}$ & $\begin{array}{c}\text { football } \\
\text { scenario 1 1 }\end{array}$ & $\begin{array}{c}\text { football } \\
\text { scenario 2 }\end{array}$ & $\begin{array}{c}\text { football } \\
\text { scenario 3 }\end{array}$ \\
\hline EDCA & 6.791392 & 4.721894 & 4.893259 & 4.086974 & 2.070394 & 1.458251 \\
\hline ICM & 3.73179 & 3.610254 & 3.734626 & 6.448081 & 4.945693 & 5.311563 \\
\hline Lin & 4.67666 & 5.575557 & 5.724116 & 4.28027 & 1.936427 & 2.392729 \\
\hline
\end{tabular}

TABLE VII. PACKET LOSS OF FOREMAN, SCENARIO 2

\begin{tabular}{l|c|c|c|c|c}
\hline & $\begin{array}{c}\text { avg } \\
\text { PSNR }\end{array}$ & $\begin{array}{c}\text { overall } \\
\text { loss }\end{array}$ & $\begin{array}{c}\text { I } \\
\text { loss }\end{array}$ & $\begin{array}{c}\text { P } \\
\text { loss }\end{array}$ & $\begin{array}{c}\text { B } \\
\text { loss }\end{array}$ \\
\hline EDCA & 25.80 & 625 & 311 & 22 & 292 \\
\hline ICM & 25.17 & 1279 & 0 & 885 & 394 \\
\hline Lin & 24.49 & 616 & 212 & 109 & 295 \\
\hline DFAA & 29.45 & 705 & 0 & 265 & 440 \\
\hline
\end{tabular}

TABLE VIII. PACKET LOSS OF FOOTBALL, SCENARIO 2

\begin{tabular}{l|c|c|c|c|c}
\hline & $\begin{array}{c}\text { avg } \\
\text { PSNR }\end{array}$ & $\begin{array}{c}\text { overall } \\
\text { loss }\end{array}$ & $\begin{array}{c}\text { I } \\
\text { loss }\end{array}$ & $\begin{array}{c}\text { P } \\
\text { loss }\end{array}$ & $\begin{array}{c}\text { B } \\
\text { loss }\end{array}$ \\
\hline EDCA & 29.09 & 496 & 182 & 280 & 34 \\
\hline ICM & 24.67 & 909 & 0 & 909 & 0 \\
\hline Lin & 28.85 & 461 & 161 & 272 & 28 \\
\hline DFAA & 30.99 & 508 & 5 & 465 & 38 \\
\hline
\end{tabular}

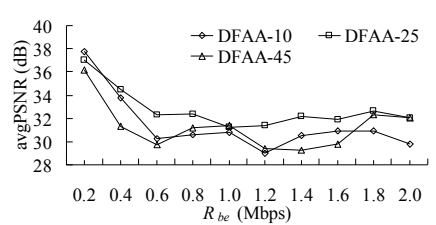

(a) foreman, $4 \mathrm{Mpbs}$ $\rightarrow$ DFAA-10 $\rightarrow$ DFAA-25

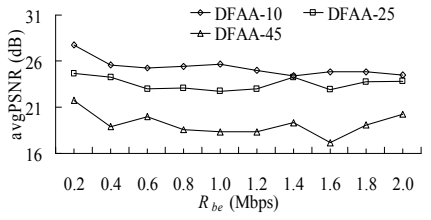

(c) foreman, $3 \mathrm{Mbps}$

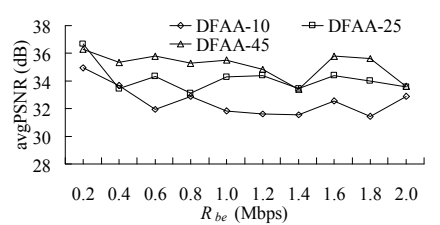

(b) football, $4 \mathrm{Mbps}$

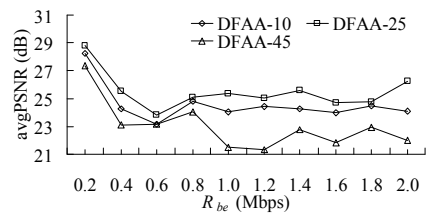

(d) football, $3 \mathrm{Mbps}$
Figure 4. Performance comparison of DFAA with different $k_{2}$

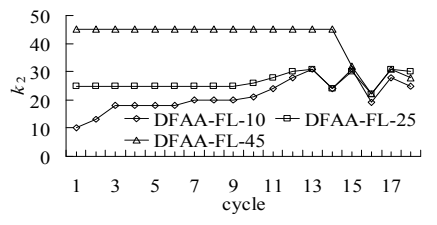

(a) foreman, $4 \mathrm{Mbps}$

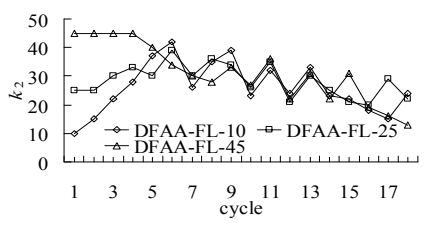

(c) foreman, $3 \mathrm{Mbps}$

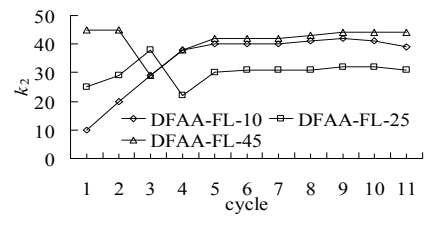

(b) football, $4 \mathrm{Mbps}$

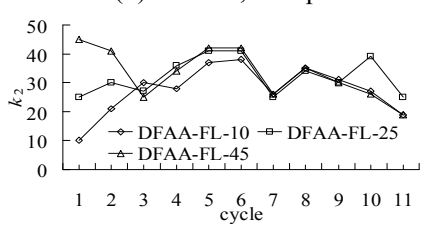

(d) football, $3 \mathrm{Mbps}$
Figure 5. Adjustment details of $k_{2}$

congestion greatly. When video sequences are transmitted in $3 \mathrm{Mbps}$ wireless links, frame loss rate remains relatively high. The FL controller has to adjust $k_{2}$ continually to find an optimal value, leading to performance degradation. It is shown that the values of $k_{2}$ in three schemes with different initial values go closer as the simulation goes on. On the contrary, most I frames and $\mathrm{P}$ frames are transmitted successfully when the link 
TABLE IX. AVERAGE PSNR VARIATION OF DFAA-FL WITH DIFFERENT INITIAL $K_{2}$

\begin{tabular}{c|c|c|c|c|c|c|c|c|c}
\hline & $\mathbf{5}$ & $\mathbf{1 0}$ & $\mathbf{1 5}$ & $\mathbf{2 0}$ & $\mathbf{2 5}$ & $\mathbf{3 0}$ & $\mathbf{3 5}$ & $\mathbf{4 0}$ & $\mathbf{4 5}$ \\
\hline foreman-4M & 32.065091 & 32.878719 & 33.161832 & 32.556565 & 32.231595 & 32.246726 & 32.611982 & 33.189742 & 33.266999 \\
\hline football-4M & 35.680533 & 35.704185 & 35.099334 & 34.895166 & 34.800419 & 34.909711 & 35.390898 & 35.933422 & 35.740335 \\
\hline foreman-3M & 23.452098 & 23.441892 & 23.133167 & 23.465348 & 23.578261 & 23.233329 & 25.082089 & 24.082125 & 24.099737 \\
\hline football-3M & 25.076173 & 25.056241 & 24.436718 & 24.659746 & 25.524935 & 25.936345 & 23.997507 & 23.865539 & 25.307283 \\
\hline
\end{tabular}

load is not heavy (4Mbps links), resulting in slight adjustment of $k_{2}$. In Fig. 5(a) after the 15th cycles and in Fig. 5(b) before the 5 th cycles, the adjustment of $k_{2}$ are remarkable because data rate of video sequence at that time produces heavy congestion.

\section{CONCLUSIONS}

In this paper we address an adaptive video scheduling scheme through wireless access networks and high speed core networks. To minimize video transmission distortion from wireless terminal to the server, adaptive scheduling mechanism should be deployed at the wireless channel for its limited bandwidth. Our proposed scheme is based on the ideas of cross layer design and unequal protection. Three enhancements are proposed in this novel scheme: (1) $D_{R}$ is defined to identify the priority of $\mathrm{AC}$ so as to reduce transmission delay of video frame; (2) $D F A A$ which considers both frame priority and network load is addressed to provide efficient unequal protection; (3) FL controllers are deployed to provide flexibility to video data rate, coding structure and network load. Simulation results show that the average PSNR of DFAA is much higher than those of EDCA, ICM and Lin in different scenarios. Moreover, FL controller can produce appropriate adjustment of DFAA parameter so that a random initialization of DFAA parameter becomes possible.

DFAA-FL is not limited to video communications over IEEE 802.11e networks. DFAA is suitable for any video codec which has limited priorities; the idea of DFAA could be used in any priority based multi-queue or single-queue unequal scheduling problem at network or MAC layer; similar FL controller(s) may be applied to other scheduling algorithms having one or more parameters to provide flexibility to environments.

For future work, we plan to consider the region-of-interest coding method to improve subjective video quality and combine multi-path routing with DFAA-FL to further enhance the performance of unequal protection.

\section{ACKNOWLEDGMENT}

This work was supported by Natural Science Foundation of China (No. 60862002, No. 60863016, No. 60963011), and Natural Science Foundation of Jiangxi Province (No. 2008GQS0015).

\section{REFERENCES}

[1] S. Kompella, S. Mao, Y. T. Hou, H. D. Sherali, "On path selection and rate allocation for video in wireless mesh networks," IEEE/ACM Transactions on Networking, vol. 17, no. 1, pp. 212-224, 2009.

[2] Ahmed Abd El Al, T. Saadawi, M. Lee, "Multi-user video streaming over multi-hop wireless networks: a distributed, cross-layer approach based on priority queuing," IEEE Journal on Selected Areas in Communications, vol. 25, no. 4, pp. 770-785, 2007.

[3] P. Navaratnam, H. Cruickshank, R. Tafazolli, "A link adaptive transport protocol for multimedia streaming applications in multi hop wireless networks," Mobile Network Applic., vol. 13, no. 3-4, pp. 246-258, 2008.

[4] M. Qin, R. Zimmermann, "Improving mobile ad-hoc streaming performance through adaptive layer selection with scalable video coding," in Proceedings of MM'07, 2007.

[5] IEEE Std 802.11e-2005, Wireless LAN medium access control (MAC) and physical layer (PHY) specifications amendment 8: medium access control (MAC) quality of service enhancements, 2005.

[6] A. Ksentini, M. Naimi, A. Guéroui, "Toward an improvement of H.264 video transmission over IEEE 802.11e through a cross-layer architecture,” IEEE Communications Magazine, 44(1): 107-114, 2006.

[7] C. H. Lin, C. K. Shieh, C. H. Ke et al. "An adaptive cross-layer mapping algorithm for MPEG-4 video transmission over IEEE 802.11e WLAN," Telecommunication Systems, vol. 42, no. 3-4, pp. 223-234, 2009.

[8] The Network Simulator - NS (version 2), http://www.isi.edu/nsnam/ns/

[9] J. Klaue, B. Rathke, A. Wolisz, "EvalVid-a framework for video transmission and quality evaluation," in Proceedings of 13th International Conference on Modelling Techniques and Tools for Computer Performance Evaluation, 2003.

[10] C. H. Ke, C. K. Shieh, W. S. Hwang, A. Ziviani, "An evaluation framework for more realistic simulations of MPEG video transmission," Journal of Information Science and Engineering, vol.24, no. 2, pp. 425$440,2008$. 